\title{
Arbeitszeitwunsch und -wirklichkeit im Familienkontext
}

\author{
Eine Analyse der Diskrepanzen zwischen präferierter und tatsächlicher Arbeitszeit
}

\section{Von Matthias Pollmann-Schult}

Zusammenfassung: Der Beitrag analysiert Diskrepanzen zwischen dem erwünschten und dem faktischen Erwerbsumfang. Im Zentrum des Interesses steht dabei die Wirkung der familialen Situation und der Erwerbskonstellation innerhalb des Haushalts auf den Wunsch zur Arbeitszeitreduktion oder -ausweitung. Die Analysen auf Basis der Daten des Sozio-oekonomischen Panels (SOEP) zeigen, dass ein beträchtlicher Anteil der Erwerbstätigen die Arbeitszeit reduzieren möchte. Bezüglich der familialen Situation werden geschlechterdivergente Effekte auf die Arbeitszeitwünsche beobachtet. Während Mütter im Vergleich zu kinderlosen Frauen eher zur Reduktion der Arbeitszeit neigen, wünschen Väter deutlich seltener eine Arbeitszeitverkürzung als Männer ohne Kinder. Ferner zeigt sich, dass bestimmte Personengruppen, die überdurchschnittlich häufig unter Zeitkonflikten leiden, wie allein erziehende Mütter und Väter in Doppelverdienerhaushalten, vergleichsweise selten ihre Arbeitszeit verkürzen möchten. Die Analysen legen nahe, dass Konflikte zwischen Beruf und Familie nicht zwangsläufig den Wunsch nach kürzeren Arbeitszeiten hervorrufen, sondern die Wirkung solcher Zeitkonflikte auf die Arbeitszeitwünsche durch moderierende Faktoren wie die ökonomischen Ressourcen des Haushalts sowie soziale Normen abgeschwächt oder verstärkt wird.

\section{Einleitung}

Wie die große Anzahl neuerer Publikationen zur ,work-life balance“ zeigt, kommt der Vereinbarkeit von Arbeit und Privatleben seit einigen Jahren eine wachsende Aufmerksamkeit zu. Dieses Thema ist nicht nur von akademischem Interesse, sondern gleichfalls für einen beträchtlichen Anteil der Erwerbstätigen von hoher Relevanz: Bei jedem fünften Beschäftigten gerät das außerberufliche Leben aufgrund der Erwerbstätigkeit häufig unter Zeitdruck (Bauer et al. 2004). Erwartungsgemäß erfahren Eltern öfter Zeitkonflikte als kinderlose Personen; so berichtet jede dritte vollzeitbeschäftigte Mutter akute Vereinbarkeitsprobleme.

Der Umstand, dass trotz der im historischen Vergleich relativ niedrigen Arbeitszeiten ein zunehmender Anteil der Erwerbstätigen Zeitkonflikte empfindet, hat verschiedene Gründe. Eine mögliche Ursache für den wachsenden Zeitdruck wird in der Einführung neuer Arbeitsund Organisationsformen gesehen, die Veränderungen der Lage und Dauer von Arbeitszeit mit sich bringt (Jürgens 2005). Mit dem sich vollziehenden Wandel vom traditionellen Modell der Normalarbeitszeit zu flexiblen Arbeitszeitmustern verbreiten sich zusehends atypische Arbeitszeiten wie Abend-, Schicht- und Wochenendarbeit, wodurch die Vereinbarkeit von Familie und Beruf erschwert wird. Zweitens hat sich das Arbeitsangebot der Haushalte in den letzten Jahrzehnten gravierend verändert. So nimmt der Anteil an Partnerschaften, in denen beide Partner erwerbstätig sind, stetig zu, wodurch sich die Zeitkonflikte verstärken, da die Gesamtzeit innerhalb einer Partnerschaft, die auf Hausarbeit und Kinderbetreuung verwendet werden kann, kontinuierlich sinkt. Drittens verzeichnen bestimmte Beschäftigtengruppen gegenwärtig eine schleichende Verlängerung der Arbeitszeiten, woraus ebenfalls Vereinbarkeitsprobleme resultieren. Zwar hat sich das durchschnittliche Arbeitsvolumen in den vergangen Jahrzehnten kaum verändert, jedoch sind Polarisierungstendenzen zu beobachten, die sich in einer Zunahme von Erwerbstätigen mit niedrigen als auch sehr hohen Arbeitszeiten äußern (Bosch 2000; Lehndorff 2003; Wagner 2001). 
Die oben skizzierten Entwicklungen lassen erwarten, dass ein erheblicher Anteil der Beschäftigten länger als eigentlich erwünscht arbeitet. Der vorliegende Beitrag geht der Frage nach, inwiefern die tatsächlichen Arbeitszeiten der Beschäftigten mit den gewünschten Arbeitszeiten übereinstimmen, wobei insbesondere der Zusammenhang zwischen familialer Situation sowie der Erwerbssituation des Haushalts einerseits und dem Wunsch zur Veränderung der Arbeitszeit andererseits beleuchtet wird. Damit zielt dieser Beitrag darauf ab, zwei bislang weitgehend unverbundene Forschungsstränge miteinander zu verknüpfen. Dies ist zum einen die arbeitsmarkt- und familiensoziologische Forschung zur Vereinbarkeit von Familie und Beruf, die häufig Zeitkonflikte konstatiert und daraus an die betriebliche Seite gerichtete Forderungen nach familienfreundlicheren als auch kürzeren Arbeitszeiten ableitet (Bauer / Munz 2005; Garhammer 2004; Rürup / Gruescu 2005), jedoch häufig die tatsächlichen Arbeitszeitwünsche der Beschäftigten unberücksichtigt lässt oder nur aggregiert betrachtet. Zum anderen untersucht die ökonomische Arbeitsmarktforschung zwar die individuelle Übereinstimmung von tatsächlicher und gewünschter Arbeitszeit, jedoch erfolgt die Ergebnisinterpretation primär in Hinblick auf die Passung von Arbeitsangebot und -nachfrage auf der Makroebene, woraus dann die Forderung nach attraktiveren Teilzeitarbeitsplätzen (Beckmann / Kempf 1996) oder einer Arbeitszeitreduktion zur Schaffung neuer Arbeitsplätze (Grözinger et al. 2008) resultiert.

Weitgehend unberücksichtigt bleiben in diesen Studien die Determinanten der Nichtübereinstimmung zwischen gewünschter und tatsächlicher Arbeitszeit. Insbesondere fehlen bislang Erkenntnisse darüber, inwiefern zeitknappe Haushaltskonstellationen den Wunsch nach kürzeren Arbeitszeiten fördern. Unabdingbar ist hier die Unterscheidung zwischen zeitbezogenen und ökonomischen Faktoren: Einerseits verstärken aus der Familien- und Erwerbssituation resultierende Zeitkonflikte den Wunsch nach kürzeren Arbeitszeiten, andererseits wird dieser Wunsch zur Arbeitszeitverkürzung durch die finanziellen Bedürfnisse des Haushalts restringiert. Die gegensätzliche Wirkung verschiedener Faktoren auf die Arbeitszeitwünsche kann dazu führen, dass bestimmte Erwerbstätige zwar erheblichen Zeitkonflikten ausgesetzt sind, aufgrund ihrer prekären finanziellen Situation ihre Arbeitszeit jedoch nicht reduzieren oder gar ausweiten möchten. Unklar ist ferner, ob Frauen und Männer in gleichartigen Kontexten ebenfalls ähnliche Arbeitszeitwünsche formulieren. Insbesondere stellt sich hier die Frage, ob geschlechtsspezifische Wirkungen der familialen Situation vorliegen. Angesichts der zumeist traditionellen Arbeitsteilung ist etwa anzunehmen, dass Väter im Vergleich zu kinderlosen Männern seltener zur Arbeitszeitverkürzung oder zur Ausweitung der Arbeitszeit neigen, wogegen bei Frauen im Zuge der Familiengründung der Wunsch zur Arbeitszeitreduzierung zunimmt.

Aus Diskrepanzen zwischen der tatsächlichen und gewünschten Arbeitszeit ergeben sich sowohl für die Erwerbstätigen als auch für die Unternehmen vielfältige negative Folgen. Erwerbstätige, die deutlich länger arbeiten als sie eigentlich möchten, erfahren nicht nur gravierende Vereinbarkeitsprobleme zwischen dem Erwerbs- und dem Privatleben (Berg et al. 2003; Moen / Dempster-McClain 1987; Reynolds 2005), sondern erleiden ebenfalls häufiger gesundheitliche Probleme (Galinsky et al. 2001). Für die Unternehmen ergeben sich vermutlich negative Produktivitätseffekte, wenn die tatsächliche Arbeitszeit signifikant von den Arbeitszeitpräferenzen der Arbeitnehmer abweicht. So verzeichnen Erwerbstätige mit Diskrepanzen zwischen tatsächlichem und präferiertem Erwerbsumfang eine geringe Betriebsbindung und eine niedrige Loyalität gegenüber dem Arbeitgeber (Stamper / Van Dyne 2001) sowie eine höhere Kündigungswahrscheinlichkeit (Böheim / Taylor 2004; Reynolds 2006).

Im folgenden Abschnitt werden zunächst theoretische Überlegungen zur Auswirkung individueller und arbeitsplatzbezogener Merkmale auf die Diskrepanz zwischen tatsächlicher und präferierter Arbeitszeit dargelegt. Hieran anschließend werden die Datenbasis und das 
methodische Vorgehen beschrieben (Abschnitt 3). Der vierte Abschnitt enthält die Ergebnisse verschiedener Modellschätzungen zum Mismatch zwischen erwünschtem und tatsächlichem Arbeitsvolumen. In einem Vorschritt werden die Folgen von Diskrepanzen zwischen der gewünschten und der tatsächlichen Arbeitszeit für das subjektive Wohlbefinden analysiert; konkret wird untersucht, inwiefern solche Diskrepanzen die Lebens- und Arbeitszufriedenheit beeinflussen. Im fünften Abschnitt werden die Ergebnisse zusammenfassend kommentiert.

\section{Determinanten der Diskrepanz zwischen erwünschtem und tatsächlichem Arbeitsvolumen}

Gemäß der Arbeitsangebotstheorie teilen Erwerbstätige die ihnen insgesamt zur Verfügung stehende Zeit nutzenmaximierend auf. Im einfachsten Fall besteht die Wahlmöglichkeit zwischen Zeit, die auf dem Arbeitsmarkt verkauft wird, und Zeit, die für Freizeit, Haus- und Familienarbeit oder Bildung verwendet wird. Sowohl die für Marktarbeit verwendete Zeit als auch die nicht auf dem Arbeitsmarkt angebotene Zeit stiften einen Nutzen. Dies ist zum einen das Erwerbseinkommen (bzw. die Güter, die hiermit erworben werden können) und zum anderen der Wert der Freizeit, der die Opportunitätskosten der Arbeit darstellt. Die präferierte Arbeitszeit der Erwerbstätigen hängt somit von dem Nutzen ab, den eine Stunde stiftet, die für Erwerbsarbeit oder Freizeit verwendet wird. Die Höhe des Nutzens einer zusätzlichen Stunde an Erwerbsarbeit wird durch private Lebensumstände wie die familiale Situation als auch Eigenschaften des Arbeitsplatzes wie die Lohnhöhe geprägt. Gemäß der Arbeitsangebotstheorie können die Arbeitnehmer ihre Arbeitsmenge frei wählen, womit die tatsächliche Arbeitszeit stets der präferierten Arbeitszeit entsprechen würde. Dieses Postulat der nichtrestringierten Arbeitszeiten ist jedoch nicht haltbar; vielmehr werden die tatsächlichen Arbeitszeiten seitens des Beschäftigungsbetriebs vorgegeben und orientieren sich an den konkreten Arbeitsanforderungen und der Arbeitsorganisation. ${ }^{1}$ Der Analyse von Arbeitszeit-Diskrepanzen liegt somit die Annahme zugrunde, dass die tatsächliche Arbeitszeit, zumindest in der kurz- und mittelfristigen Betrachtung, nicht variiert werden kann. Diskrepanzen zwischen der erwünschten und der faktischen Arbeitszeit entstehen dann, wenn Arbeitgeber und Arbeitnehmer unterschiedliche Präferenzen hinsichtlich des Arbeitsvolumens aufweisen.

Die erwünschte Arbeitszeit und damit auch das Ausmaß der Diskrepanz zwischen tatsächlicher und präferierter Arbeitszeit werden zum einen durch die familiale Situation geprägt. Das Arbeitsangebot der Erwerbstätigen hängt stark davon ab, wie viel Reproduktionsarbeit, also Hausarbeit und Kinderbetreuung, ein Haushalt zu leisten hat und wie die Mitglieder sie untereinander aufteilen (Bielenski et al. 2002). Im Zuge der Familiengründung bildet sich zumeist ein traditionelles Arrangement der Arbeitsteilung heraus, bei der sich die Frau primär um die Hausarbeit und Kinderbetreuung kümmert und der Mann sich auf die Erwerbsarbeit fokussiert (Schulz / Blossfeld 2006). Da die Familienarbeit zumeist der Frau zugewiesen wird, ist anzunehmen, dass Mütter ihre Arbeitszeit eher reduzieren möchten als kinderlose Frauen mit vergleichbaren Arbeitszeiten. Analog ist zu erwarten, dass Väter nach der Familiengründung ihre Arbeitszeit ausdehnen möchten, um den Verdienstausfall der Partnerin zu kompensieren. Bisherige Studien zum Arbeitsmarktverhalten von Vätern finden zwar keine nennenswerten Veränderungen der Arbeitszeit im Zuge der Familiengründung (Hamermesh 1996; Pollmann-Schult / Diewald 2007; Vaskovics et al. 2000), jedoch bleibt unklar, ob sich das Arbeitsangebot der Väter tatsächlich nicht ändert, oder aber ob mögliche Änderungswünsche aufgrund rigider Arbeitszeitvorgaben nicht realisiert werden können.

1 Verschiedene empirische Studien (etwa Böheim / Taylor 2004) zeigen, dass Änderungen der tatsächlichen Arbeitszeit eher durch zwischenbetriebliche Stellenwechsel denn betriebsinterne Wechsel oder „on-the-job“ realisiert werden. 
Ein weiterer Faktor, der das Arbeitsangebot und damit ebenfalls den Wunsch zur Arbeitszeitreduktion oder -ausweitung prägen dürfte, ist das Erwerbsverhalten des Partners bzw. der Partnerin (siehe Drago et al. 2005; Jacobs / Gerson 2001; Jacobs / Gerson 2004). Allgemein wird davon ausgegangen, dass Individuen in Paarhaushalten ihr Arbeitsangebot nicht voneinander unabhängig bestimmen, sondern das Arbeitsangebot des einen Partners das des anderen beeinflusst (Lundberg 1988). Hier zeigt sich, dass insbesondere das Arbeitsangebot von Frauen durch die Erwerbssituation ihrer Partner geprägt wird, wobei mit steigenden Einkommenschancen des Mannes das Arbeitsangebot der Frau sinkt (Blau / Kahn 2007; Devereux 2004; Morissette / Hou 2008). Das Erwerbseinkommen der Ehefrau oder Partnerin hat dagegen einen deutlich geringen Effekt auf das Arbeitsangebot des Mannes (Blau / Kahn 2007). Somit ist eine substituierende Beziehung des Arbeitsangebots im Paarkontext zu erwarten, wobei mit zunehmender Arbeitszeit des einen Partners der gewünschte Erwerbsumfang des anderen Partners sinkt. Wir nehmen daher an, dass Frauen und Männer in Doppelverdienerhaushalten eher zu einer Reduktion und seltener zu einer Ausweitung der Arbeitszeit neigen als Personen in Einverdienerhaushalten. Im Gegensatz zu Paaren mit Kindern sind kinderlose Haushalte, bei denen das Arbeitsangebot nicht durch die notwendige Familienarbeit eingeschränkt wird, wesentlich flexibler in der Gestaltung ihrer Arbeitszeiten. Daher sollten die Auswirkungen des Erwerbsstatus des Partners auf das Arbeitsangebot bei kinderlosen Paaren deutlich schwächer ausfallen als bei Müttern und Vätern.

Ferner ist ein positiver Zusammenhang zwischen dem Bildungsniveau und der Neigung zur Arbeitszeitreduktion zu erwarten. Akademiker üben überproportional häufig gut bezahlte Tätigkeiten mit hohen Arbeitsbelastungen aus; beide Faktoren dürften einen positiven Effekt auf die Bereitschaft zur Arbeitsverkürzung haben (siehe unten). Schließlich erwarten wir, dass die Neigung zur Reduktion der Arbeitszeit im Berufsverlauf zu- und die zur Arbeitszeitausdehnung abnimmt. Diese Annahme basiert auf der empirischen Beobachtung eines mit dem Alter sinkenden präferierten Erwerbsumfangs (Bielenski et al. 2002), wodurch sich der Wunsch zur Verlängerung bzw. Verkürzung der Arbeitszeit entsprechend verschieben sollte.

Neben individuellen Charakteristika haben ebenfalls Arbeitsplatzmerkmale Einfluss auf die präferierten Arbeitszeiten. Gemäß der Arbeitsangebotstheorie wird das gewünschte Arbeitsvolumen wesentlich durch die Einkommenshöhe geprägt. Hier ist ein so genannter Einkommenseffekt zu erwarten, wonach Erwerbstätige mit steigendem Reallohn ihr Arbeitsangebot reduzieren, da das gleiche Einkommen in einer kürzeren Arbeitszeit erzielt werden kann (Franz 2003). Inwiefern sich die Arbeitszeitwünsche tatsächlich realisieren lassen, ist vor allem von der Arbeitszeitregelung und der Arbeitszeitflexibilität des Beschäftigungsbetriebs anhängig. Allgemein bieten Großbetriebe häufiger als kleinere Betriebe familienfreundliche Arbeitszeitmodelle an, die die Vereinbarkeit von Beruf und Familie erleichtern (Glass / Estes 1997). Folglich ist anzunehmen, dass Beschäftigte in größeren Unternehmen eine höhere Passung von tatsächlicher und gewünschter Arbeitszeit aufweisen. Unterschiede in der Arbeitszeitflexibilität sind ebenfalls zwischen Beschäftigten im öffentlichen Dienst und in der Privatwirtschaft vorhanden. Die Arbeitszeitregelung im öffentlichen Dienst ist oftmals weniger rigide und ein Wechsel in die Teilzeitbeschäftigung leichter möglich als in der Privatwirtschaft, womit Erwerbstätige im öffentlichen Dienst ihre präferieren Arbeitszeiten besser realisieren können und seltener zur Arbeitszeitverkürzung neigen sollten. Ferner ist anzunehmen, dass Beschäftigte im hochqualifizierten Bereich, die oftmals besonders stark gefordert werden und deren Arbeitszeit durch hohe Arbeitsanforderungen weitgehend vorgegeben ist, häufiger einen Mismatch hinsichtlich der Arbeitszeit berichten als andere Beschäftigte.

Selbstständig Erwerbstätige unterscheiden sich von abhängig Beschäftigten hinsichtlich der Arbeitszeit dahingehend, dass Selbstständige in weitaus geringerem Maße institutionel- 
len Restriktionen unterworfen sind. Demnach sollten Selbstständige in höherem Maße ihre gewünschte Arbeitszeit realisieren können als abhängig Beschäftigte und insbesondere seltener den Wunsch nach einer Arbeitsreduktion äußern. Ferner ist zu erwarten, dass die tatsächliche Arbeitszeit selbst einen starken Effekt auf die Diskrepanz zwischen erwünschter und faktischer Arbeitszeit hat. Ein Großteil der Beschäftigten präferiert ein Arbeitsvolumen zwischen 30 und 40 Wochenstunden (Bielenski et al. 2002; Bosch 2000). Demgemäß ist anzunehmen, dass Erwerbstätige mit langen Arbeitszeiten zu einer Verringerung sowie Personen mit einem eher geringen Arbeitsvolumen zu einer Ausdehnung der Arbeitszeit neigen.

Neben den geschilderten individuellen und arbeitsplatzbezogenen Faktoren dürften die wirtschaftlichen Rahmenbedingungen das Ausmaß der Übereinstimmung von erwünschter und tatsächlicher Arbeitszeit beeinflussen. Hier ist zu erwarten, dass viele Beschäftigte in Phasen hoher Arbeitslosigkeit aus Furcht vor einem Arbeitsplatzverlust eher zu einer Arbeitszeitausweitung und seltener zu einer Verringerung des Arbeitsvolumens neigen.

\section{Daten und Methode}

\section{Datenbasis und Variablen}

Die Datenbasis der Analysen ist das Sozio-oekonomische Panel (Wagner et al. 2007). Das SOEP ist eine seit 1984 in Westdeutschland und ab 1990 ebenfalls in den neuen Bundesländern durchgeführte Wiederholungsbefragung repräsentativer Haushalte. Dieser Datensatz enthält detaillierte Informationen zum tatsächlichen sowie präferierten Erwerbsumfang und eignet sich daher sehr gut zur Untersuchung der hier präsentierten Fragestellung. Für die folgenden Analysen werden die Daten der Jahre 1985 bis 2005 (Wellen B-V) verwendet. ${ }^{2}$ Berücksichtigt werden erwerbstätige Personen zwischen 18 und 60 Jahren. Personen in Ausbildung bleiben von der Analyse unberücksichtigt. Die Analyse beschränkt sich ferner auf die alten Bundesländer, da sich die Arbeitszeitpräferenzen sowie die Wechselwirkungen von Erwerbstätigkeit und familialen Prozessen in den neuen Bundesländern deutlich von denen in den alten Ländern unterscheiden dürften. Nach Anwendung der Selektionskriterien stehen für die Analyse 123.321 Personenjahre von 22.369 Personen zur Verfügung.

Zur Messung der Diskrepanz zwischen dem präferierten und dem faktischen Arbeitsvolumen werden die im SOEP enthaltenen Informationen zur tatsächlichen und gewünschten Arbeitszeit genutzt. Bei der Nennung der erwünschten Arbeitszeit sollen die Respondenten bedenken, dass sich ihr Einkommen entsprechend ihrer Wunscharbeitszeit erhöhen oder vermindern würde. ${ }^{3}$ In Anlehnung an Baaijens et al. (2005) gehen wir von einem Mismatch zwischen der erwünschten und tatsächlichen Arbeitszeit aus, wenn das präferierte Arbeitsvolumen um mindestens vier Wochenstunden von dem faktischen Volumen abweicht. Somit lassen sich drei Gruppen von Beschäftigten unterscheiden: Personen, die mehr arbeiten als sie eigentlich möchten und folglich ihre Arbeitszeit reduzieren wollen, Personen, bei denen erwünschte und tatsächliche Arbeitszeit übereinstimmen und Personen, die weniger als erwünscht arbeiten und folglich ihre Arbeitszeit ausweiten möchten.

In einem ersten Analyseschritt werden die Auswirkungen von Diskrepanzen zwischen erwünschtem und tatsächlichem Erwerbsumfang auf die Lebens- sowie die Arbeitszufriedenheit untersucht. Die Lebenszufriedenheit wird im SOEP mit folgender Frage erhoben: „Wie zufrieden sind Sie gegenwärtig, alles in allem, mit Ihrem Leben“. Hinsichtlich der Zufrieden-

2 Die Daten der Wellen A (1984) und M (1996) werden nicht genutzt, da die gewünschte Arbeitszeit in diesen Jahren nicht erhoben wurde.

3 Hier wird unterstellt, dass die Arbeitnehmer ihre Arbeitszeit bei gleich bleibendem Stundenlohn ändern können. Dies ist jedoch häufig nicht der Fall, da etwa der Wechsel auf eine Teilzeitstelle dauerhaft die Karrierechancen verringert und damit langfristig einen niedrigeren Stundenlohn nach sich zieht. 
heit mit der Erwerbstätigkeit wird gefragt: „Wie zufrieden sind Sie mit Ihrer Arbeit?“ Die Lebens- und Arbeitszufriedenheit wurde anhand einer 11-stufigen Skala von $0=$ ganz und gar unzufrieden bis $10=$ ganz und gar zufrieden erhoben.

Eine zentrale Kovariate ist die Familiensituation, die hier über das Zusammenwohnen mit einem Partner/einer Partnerin und das Alter des jüngsten Kindes erfasst wird. Wir unterscheiden zwischen kinderlosen Personen ohne Partner, verheirateten Personen bzw. Personen in einer nichtehelichen Partnerschaft ohne Kinder, ferner zwischen Personen, deren jüngstes Kind unter sieben Jahren alt ist, Personen, deren jüngstes Kind zwischen sieben und 17 Jahren alt ist, sowie Personen mit volljährigen Kindern. Weiterhin werden allein erziehende Frauen mit Kindern unter 18 Jahren berücksichtigt, nicht aber allein erziehende Väter. ${ }^{4}$ Die Referenzkategorie in den multivariaten Analysen bilden kinderlose Frauen bzw. Männer in einer Partnerschaft.

Als weitere Kovariaten werden in den multivariaten Analysen Alter, Qualifikationsniveau (kein Ausbildungsabschluss, Berufsausbildung, Fach- / Hochschulabschluss), tatsächliche Wochenarbeitszeit (1-14 Stunden, 15-29 Stunden, 30-45 Stunden, über 45 Stunden), Bruttoerwerbseinkommen (logarithmiert ${ }^{5}$ und deflationiert zum Basisjahr 2000), Berufsposition (hochqualifizierte Tätigkeiten und Tätigkeiten mit umfassenden Führungsaufgaben vs. andere), Beschäftigung im öffentlichen Dienst, Betriebsgröße (bis 20 Mitarbeiter, 20-199 Mitarbeiter, 200-1999 Mitarbeiter, 2000 und mehr Mitarbeiter) und die jährliche Arbeitslosenquote auf Ebene der einzelnen Bundesländer berücksichtigt. Da die Effekte von familialer Situation und Geschlecht auf die Diskrepanz zwischen erwünschtem und faktischem Arbeitsvolumen stark interagieren dürften, werden alle Berechnungen getrennt für Frauen und Männer durchgeführt.

Um für Veränderungen im Zeitverlauf zu kontrollieren, werden zusätzlich Dummy-Variablen, die das jeweilige Befragungsjahr abbilden, in die Modelle aufgenommen. Die Koeffizienten dieser Dummy-Variablen werden in den Tabellen jedoch nicht ausgewiesen. ${ }^{6}$

\section{Analyseverfahren}

Die Analyse des Mitmatches im Arbeitsvolumen erfolgt anhand von Random-Effects Logit Regressionen ${ }^{7}$ (siehe zu diesem Verfahren Wooldridge 2000). Hierbei handelt es sich um ein Regressionsverfahren für Paneldaten mit einer binären abhängigen Variablen. Der Vorteil der Panelanalyse besteht insbesondere darin, dass für unbeobachtete Heterogenität kontrolliert werden kann. Unbeobachtete Heterogenität der Respondenten führt in der Regel zu einer Verzerrung der Schätzergebnisse, wenn diese Eigenschaften mit der anhängigen Variablen als auch mit den unabhängigen Variablen korrelieren. In Bezug auf die hier zu untersuchende Fragestellung ist denkbar, dass unbeobachtete Eigenschaften wie die Karriereorien-

4 Im verwendeten Datensatz konnten praktisch keine allein erziehenden Väter identifiziert werden, so dass diese Kategorie wegfällt.

5 Da vorherige Analyseschritte einen kurvlinearen Effekt des Einkommens die Wahrscheinlichkeit eines Mismatches hinsichtlich der Arbeitszeit aufgezeigt haben, wird das Bruttoeinkommen nicht in linearer, sondern in logarithmierter Form berücksichtigt.

6 Über den Zeitraum sind keine systematischen Veränderungen des Mismatches hinsichtlich der Arbeitszeit beobachtbar.

7 Eine Alternative zur Random-Effects Regression stellt die Fixed-Effects Regression dar. Dieses Verfahren hat bei der Analyse binärer abhängiger Variablen jedoch den entscheidenden Nachteil, dass alle Fälle aus der Analyse ausgeschlossen werden müssen, bei denen die Responsevariable zeitkonstant ist. Aus diesem Grund werden in der Regel Random-Effects Logit Regressionen gegenüber FixedEffects Logit Regressionen bevorzugt (Madalla 1987). 
tierung der Befragungspersonen sowohl mit der gewünschten Arbeitszeit als auch mit der familialen Situation korrelieren.

Vereinfacht formuliert unterscheidet sich die Random-Effects Regression von der OLSRegression dahingehend, dass die abhängige Variable sowie die unabhängigen Variablen um den individuellen Mittelwert bereinigt werden, indem jeweils die Differenz zwischen dem aktuellen Wert einer Variablen zu einem bestimmten Zeitpunkt und dem Mittelwert für diese Person über alle beobachteten Zeitpunkte berechnet wird. Bei der Random-EffectsSchätzung wird sowohl die Variation der Beobachtungen um den jeweiligen Mittelwert als auch die Variation dieser Mittelwerte um dem Gesamtmittelwert berücksichtigt. Der Fehlerterm wird in zwei Komponenten zerlegt, einen zeitpunktbezogenen idiosynkratischen Fehler und einen zeitkonstanten unbeobachteten Faktor, wobei der letztere Faktor als Zufallsvariable geschätzt wird. Anhand dieser Vorgehensweise wird die unbeobachtete Heterogenität minimiert. Die Interpretation der Koeffizienten entspricht der einer gewöhnlichen logistischen Regression.

\section{Empirische Ergebnisse}

\section{Arbeitszeit-Mismatch und subjektives Wohlbefinden}

In einem ersten Schritt werden die Auswirkungen einer Nichtübereinstimmung zwischen präferierter und tatsächlicher Arbeitszeit auf das subjektive Wohlbefinden untersucht. Hierzu wird zunächst in Tabelle 1 der Anteil der Personen ausgewiesen, die ihre gegenwärtige Lebens- bzw. Arbeitszufriedenheit mit 8-10 auf einer Skala von 0 bis 10 bewerten und somit als zufrieden bzw. sehr zufrieden bezeichnet werden können. Wie ersichtlich ist, sind Erwerbstätige, bei denen erwünschte und faktische Arbeitszeit übereinstimmen, deutlich zufriedener als Personen, die entweder länger oder aber kürzer arbeiten als sie eigentlich möchten. So sind beispielsweise $54 \%$ der Frauen ohne einen Mismatch hinsichtlich der Arbeitszeit, aber nur $48 \%$ der weiblichen Erwerbstätigen, die ihre Arbeitszeit reduzieren wollen, mit ihrem gegenwärtigen Leben zufrieden oder sehr zufrieden. Ferner zeigt sich, dass unterbeschäftigte Erwerbstätige eine deutlich geringere Lebenszufriedenheit berichten als überbeschäftigte Personen.

Tabelle 1: Anteil der Erwerbstätigen, die mit ihrem gegenwärtigem Leben bzw. ihrer Arbeit zufrieden/sehr zufrieden sind

\begin{tabular}{lcccc} 
& \multicolumn{2}{c}{ Lebenszufriedenheit } & \multicolumn{2}{c}{ Arbeitszufriedenheit } \\
& Frauen & Männer & Frauen & Männer \\
\hline Erwünschte = tatsächliche Arbeitszeit & 54 & 52 & 54 & 54 \\
Arbeitszeitreduktion erwünscht & 48 & 49 & 47 & 50 \\
Arbeitszeitausweitung erwünscht & 40 & 39 & 44 & 45
\end{tabular}

Zufrieden/sehr zufrieden $=$ Werte 8-10 auf einer Skala von 0 bis 10 .

Quelle: SOEP 1985-2006, eigene Berechnungen.

Ein sehr ähnliches Ergebnismuster ergibt sich bei der Betrachtung der Arbeitszufriedenheit. Auch hier findet sich bei Erwerbstätigen ohne Arbeitszeit-Mismatch der höchste Anteil und unter den Erwerbstätigen mit dem Wunsch zur Arbeitszeitausweitung der niedrigste Anteil an zufriedenen bzw. sehr zufriedenen Personen.

Die in Tabelle 1 ausgewiesenen Befunde erlauben jedoch keine Rückschlüsse auf die Kausalrichtung des beobachteten Zusammenhangs. Die niedrigere Zufriedenheit bei Personen mit Diskrepanzen zwischen erwünschter und tatsächlicher Arbeitszeit bedeutet nicht zwangsläufig, dass sich eine solche Nichtübereinstimmung negativ auf die Lebens- oder Arbeitszufriedenheit auswirkt. Ebenfalls ist eine umgekehrte Kausalwirkung denkbar, indem Personen 
mit einer niedrigen Lebenszufriedenheit überproportional häufig Beschäftigungsprobleme erfahren und daher Schwierigkeiten haben, einen ihren Wünschen entsprechenden Arbeitsplatz zu finden. Gleichfalls kann eine niedrige Arbeitszufriedenheit den Wunsch zur Arbeitszeitreduktion hervorrufen. Eine Analyse des Effekts des Arbeitszeit-Mismatches auf die beiden betrachteten Zufriedenheitsdimensionen erfordert eine Längsschnittbetrachtung. Im Folgenden wird dieser Effekt anhand einer Random-Effects Panelregression untersucht, wobei für die geschilderten Selektionseffekte kontrolliert wird. Als Referenzgruppe fungieren Personen, bei denen erwünschte und tatsächliche Arbeitszeit übereinstimmen.

Tabelle 2: Determinanten der Lebens- und Arbeitszufriedenheit (Random-Effects Regressionen)

\begin{tabular}{lcccc} 
& \multicolumn{2}{c}{ Lebenszufriedenheit } & \multicolumn{2}{c}{ Arbeitszufriedenheit } \\
& Frauen & Männer & Frauen & Männer \\
\hline Erwünschte $=$ tatsächliche AZ & - & - & - & - \\
Arbeitszeitreduktion erwünscht & $-0,1119^{* *}$ & $-0,1157^{* *}$ & $-0,3207^{* *}$ & $-0,2536^{* *}$ \\
Arbeitszeitausweitung erwünscht & $-0,2532^{* *}$ & $-0,2375^{* *}$ & $-0,2480^{* *}$ & $-0,1940^{* *}$ \\
& & & & \\
& & & & \\
& Signifikanzniveau: ** $=\mathrm{p}<0,01 ; *=\mathrm{p}<0,05 ;^{+}=\mathrm{p}<0,1$. & & \\
Fallzahl (Personenjahr) & 49.293 & 70.677 & 48.401 & 69.881 \\
$\mathrm{R}^{2}$ & 0,03 & 0,03 & 0,02 & 0,03
\end{tabular}

Kontrolliert wird für: Familiensituation, Alter, Qualifikationsniveau, tatsächliche Arbeitszeit, Bruttomonatseinkommen, hochqualifizierte Tätigkeit, Beschäftigung im öffentlichen Dienst, Betriebsgröße, Arbeitslosenquote.

Quelle: SOEP 1985-2006, eigene Berechnungen.

Wie die in Tabelle 2 dargestellten Ergebnisse verdeutlichen, hat eine Nichtübereinstimmung zwischen dem gewünschten und dem faktischen Erwerbsumfang in der Tat signifikant negative Auswirkungen auf die Lebens- und Arbeitszufriedenheit. Im Gegensatz zu den deskriptiven Befunden deuten die multivariaten Ergebnisse allerdings darauf hin, dass Überbeschäftigte mit ihrer Arbeit unzufriedener sind als Unterbeschäftigte, wogegen Unterbeschäftigte eine niedrigere Lebenszufriedenheit verzeichnen als Überbeschäftigte.

\section{Determinanten der Diskrepanzen zwischen tatsächlicher und erwünschter Arbeitszeit}

Zunächst präsentieren wir einige deskriptive Befunde zum Ausmaß der Nichtübereinstimmung zwischen erwünschter und tatsächlicher Arbeitszeit. Hieran anschließend wird der Einfluss individueller sowie arbeitsplatz- und betriebsbezogener Faktoren auf die Wahrscheinlichkeit des Mismatches hinsichtlich der Arbeitszeit multivariat untersucht. In einem zweiten multivariaten Analyseschritt wird zusätzlich der Erwerbsstatus des Partners bzw. der Partnerin berücksichtigt.

Die durchschnittliche erwünschte und tatsächliche Arbeitszeit der abhängig Beschäftigten sind in Tabelle 3 ausgewiesen. Wie ersichtlich ist, liegt die gewünschte Arbeitszeit bei allen betrachteten Gruppen unter dem faktischen Arbeitsvolumen. Die größte Abweichung zwischen präferierter und tatsächlicher Arbeitszeit ist bei kinderlosen Frauen in Partnerschaft zu beobachten; hier beträgt die mittlere Differenz 5,3 Stunden. Die größte Übereinstimmung verzeichnen Mütter mit Kindern im Vorschulalter, bei denen die tatsächliche Arbeitszeit die erwünschte durchschnittlich um 2 Wochenstunden übersteigt. 
Tabelle 3: Erwünschte und tatsächliche Arbeitszeit in Wochenstunden

\begin{tabular}{lcccc} 
& \multicolumn{2}{c}{ Männer } & \multicolumn{2}{c}{ Frauen } \\
& $\begin{array}{c}\text { Erwünschte } \\
\text { Arbeitszeit }\end{array}$ & $\begin{array}{c}\text { Tatsächliche } \\
\text { Arbeitszeit }\end{array}$ & $\begin{array}{c}\text { Erwünschte } \\
\text { Arbeitszeit }\end{array}$ & $\begin{array}{c}\text { Tatsächliche } \\
\text { Arbeitszeit }\end{array}$ \\
\hline Kein/e Partner/in & 38,4 & 42,6 & 35,7 & 39,8 \\
Mit Partner/in, keine Kinder & 38,4 & 43,6 & 33,3 & 38,6 \\
Mit Partner/in, Kind unter 7 Jah- & 39,6 & 44,4 & 25,0 & 27,0 \\
ren & 39,5 & 44,6 & 26,2 & 28,4 \\
Mit Partner/in, Kind 7-17 Jahre & - & - & 32,4 & 34,5 \\
Allein erziehend & 39,2 & 44,4 & 29,4 & 32,7 \\
Kind über 17 Jahre & & &
\end{tabular}

Quelle: SOEP 1985-2006, eigene Berechnungen.

Wie erwartet wurde, wirkt sich die familiale Situation sehr unterschiedlich auf die Arbeitszeitpräferenzen und das tatsächliche Arbeitsvolumen von Frauen und Männern aus. Das Arbeitsangebot der Männer scheint im Zuge der Familiengründung leicht zuzunehmen. So berichten Väter eine um eine Wochenstunde höhere gewünschte sowie tatsächliche Arbeitszeit als kinderlose Männer in Partnerschaft. Dagegen nimmt das tatsächliche als auch das präferierte Arbeitsvolumen bei Frauen im Zuge der Familiengründung stark ab, wobei Mütter mit Kindern im Vorschulalter die kürzeste Arbeitszeit als auch das niedrigste erwünschte Arbeitsvolumen berichten. Beachtenswert ist die hohe gewünschte Arbeitszeit der allein erziehenden Frauen, die unterhalb dem präferieren Arbeitsvolumen der kinderlosen Frauen, aber deutlich über dem der Mütter in einer Partnerschaft liegt. Offensichtlich wird das Arbeitsangebot der allein Erziehenden stärker durch finanzielle Notwendigkeiten als durch die familiären Zeitbedürfnisse geprägt.

Die in Tabelle 3 dargestellten Durchschnittswerte geben keine Auskunft über den Anteil der Erwerbstätigen, die ihre Arbeitszeit reduzieren oder ausdehnen möchten. Diese Anteilswerte sind in Tabelle 4 ausgewiesen. Hier zeigt sich, dass lediglich bei etwa jedem zweiten Beschäftigten tatsächliche und erwünschte Arbeitszeit übereinstimmen. Die Mehrheit der Beschäftigten mit einer Diskrepanz zwischen präferiertem und faktischem Arbeitsvolumen arbeitet länger als gewünscht und möchte die Arbeitszeit reduzieren. Auch in Tabelle 4 ist bei Männern nur ein schwacher Zusammenhang zwischen der familialen Situation und der Wahrscheinlichkeit eines Mismatches bezüglich der Arbeitszeit ersichtlich. Bemerkenswert ist allerdings, dass partnerlose Männer die höchste Übereinstimmung zwischen erwünschter und tatsächlicher Arbeitszeit berichten, was wahrscheinlich auf die Tatsache zurückzuführen ist, dass diese Personen ihr Arbeitsangebot frei von partnerschaftlichen und familialen Verpflichtungen festlegen können. 
Tabelle 4: Anteil der Erwerbstätigen mit einer Diskrepanz zwischen gewünschter und tatsächlicher Arbeitszeit

\begin{tabular}{lcccc} 
& \multicolumn{2}{c}{ Männer } & \multicolumn{2}{c}{ Frauen } \\
& $\begin{array}{c}\text { Arbeitszeitre- } \\
\text { duktion } \\
\text { erwünscht }\end{array}$ & $\begin{array}{c}\text { Arbeitszeitaus- } \\
\text { weitung } \\
\text { erwünscht }\end{array}$ & $\begin{array}{c}\text { Arbeitszeitre- } \\
\text { duktion } \\
\text { erwünscht }\end{array}$ & $\begin{array}{c}\text { Arbeitszeitaus- } \\
\text { weitung } \\
\text { erwünscht }\end{array}$ \\
\hline Kein/e Partner/in & 43,8 & 8,5 & 45,6 & 6,9 \\
Mit Partner/in, keine Kinder & 50,1 & 6,5 & 52,5 & 6,2 \\
Mit Partner/in, Kind unter 7 Jah- & 48,8 & 5,9 & 31,5 & 15,3 \\
ren & 48,7 & 5,6 & 32,6 & 15,7 \\
Mit Partner/in, Kind 7-17 Jahre & - & - & 34,6 & 17,1 \\
Allein erziehend & 48,5 & 4,7 & 37,5 & 9,7 \\
Kind über 17 Jahre & & &
\end{tabular}

Quelle: SOEP 1985-2006, eigene Berechnungen.

Bei Frauen variiert der Wunsch zur Arbeitszeitreduzierung oder -ausweitung stark mit der familialen Situation. Während kinderlose Frauen oftmals ihren Erwerbsumfang reduzieren möchten, streben Mütter vergleichsweise häufig eine Verlängerung ihrer Arbeitszeit an. So äußern nur etwa $7 \%$ der kinderlosen Frauen, aber $15 \%$ der Mütter den Wunsch zur Arbeitszeitausweitung. Gerade allein erziehende Mütter, die sich oftmals in einer ökonomisch prekären Lage befinden und ein hohes Armutsrisiko aufweisen, neigen relativ häufig zur Ausdehnung des Erwerbsumfangs. Anscheinend erfolgt die Arbeitszeitreduktion von Frauen nach der Familiengründung oftmals nicht freiwillig, sondern ist unzureichenden Möglichkeiten der Kinderbetreuung geschuldet. Der Befund, dass Frauen mit Kindern vergleichsweise selten ihre Arbeitszeit reduzieren möchten, widerspricht nicht zwangsläufig der oben formulierten Annahme, wonach Mütter eher zu einer Arbeitszeitreduktion neigen sollten als kinderlose Frauen mit vergleichbaren Arbeitszeiten. Angesichts des geringeren Erwerbsumfangs der Mütter dürften so genannte „Floor Effekte“ vorliegen. Dies bedeutet, dass Mütter aufgrund ihrer bereits niedrigen Arbeitszeiten eher zu einer Arbeitszeitverlängerung und seltener zu einer Arbeitszeitverkürzung neigen als Personen mit einem durchschnittlichen Erwerbsumfang. Diese Verzerrungen lassen sich in den multivariaten Analysen eliminieren, indem für die tatsächliche Arbeitszeit kontrolliert wird.

Im Folgenden werden die Determinanten eines Mismatches jeweils getrennt für Männer und Frauen anhand von Random-Effects Logit Regressionen untersucht (Tabelle 5). Bemerkenswert ist erst einmal der Befund, dass persönliche Charakteristika und Arbeitsplatzeigenschaften auch dann einen Effekt auf den Arbeitszeit-Mismatch haben, wenn für die objektive Arbeitsbelastung in Form des tatsächlichen Arbeitsvolumens kontrolliert wird. Offenbar wird die subjektive Wahrnehmung der Arbeitsbelastung und der daraus resultierende Wunsch zur Arbeitszeitreduktion oder -ausweitung erheblich durch persönliche als auch betriebliche Merkmale beeinflusst. Die in Tabelle 5 ausgewiesenen Ergebnisse deuten darauf hin, dass partnerlose Männer signifikant seltener ihre Arbeitszeit ausdehnen sowie reduzieren möchten als kinderlose Männer mit einer Partnerin und damit eine höhere Übereinstimmung zwischen der erwünschten und tatsächlichen Arbeitszeit verzeichnen. Dies mag, wie schon oben erwähnt, darauf zurückzuführen sein, dass Männer ohne Partnerin ihre Arbeitszeiten freier bestimmen können als Männer in einer Partnerschaft. 
Tabelle 5: Determinanten einer gewünschten Arbeitszeitreduktion / Arbeitszeitausweitung (Random-Effects Logit Regression)

\begin{tabular}{|c|c|c|c|c|}
\hline & \multicolumn{2}{|c|}{ Männer } & \multicolumn{2}{|c|}{ Frauen } \\
\hline & $\begin{array}{l}\text { Arbeitszeitre- } \\
\text { duktion } \\
\text { erwünscht }\end{array}$ & $\begin{array}{l}\text { Arbeitszeitaus- } \\
\text { weitung } \\
\text { erwünscht }\end{array}$ & $\begin{array}{l}\text { Arbeitszeitre- } \\
\text { duktion } \\
\text { erwünscht }\end{array}$ & $\begin{array}{l}\text { Arbeitszeitaus- } \\
\text { weitung } \\
\text { erwünscht }\end{array}$ \\
\hline Kein/e Partner/in & $-0,1838^{* *}$ & $-0,2473 * *$ & $-0,4571 * *$ & $0,2075^{*}$ \\
\hline Kind unter 7 Jahren & $-0,2142 * *$ & $-0,0311$ & $0,2008 * *$ & $-0,5867 * *$ \\
\hline Kind 7-17 Jahre & $-0,2198^{* *}$ & $-0,0319$ & 0,0977 & $-0,1934 *$ \\
\hline Allein erziehend & - & - & $-0,5606 * *$ & $0,8406 * *$ \\
\hline Kind über 17 Jahre & $-0,1619 * *$ & $-0,1330$ & $-0,1482 *$ & 0,0566 \\
\hline Alter in Jahren & 0,0006 & $-0,0285^{* *}$ & $0,0112 * *$ & $-0,0367 * *$ \\
\hline Berufsausbildung & $0,2816 * *$ & 0,0529 & $0,4765 * *$ & $-0,0019$ \\
\hline Hochschulabschluss & $0,5788 * *$ & 0,0777 & $0,8866 * *$ & 0,1449 \\
\hline Arbeitszeit: bis 14 Stunden & $-4,1279 * *$ & $4,1934 * *$ & $-4,5659 * *$ & $3,9022 * *$ \\
\hline Arbeitszeit: 15-29 Stunden & $-1,8884 * *$ & 3,4848 & $-1,9623 * *$ & $2,6446 * *$ \\
\hline Arbeitszeit: über 45 Stunden & $2,6673 * *$ & $-1,6360$ & $2,7394 * *$ & $-1,5096 * *$ \\
\hline Log. Bruttoeinkommen & $0,4000 * *$ & $-0,3805 * *$ & $0,3596 * *$ & $-0,7053^{* *}$ \\
\hline Hochqualifizierte Tätigkeit & $0,3146 * *$ & $-0,1777^{+}$ & $0,1430 *$ & $-0,0333$ \\
\hline Öffentlicher Dienst & $0,0823 *$ & $-0,1938 *$ & $-0,0817^{+}$ & 0,0968 \\
\hline Betriebsgröße bis $20 \mathrm{MA}$ & 0,0625 & $-0,0618$ & 0,0725 & $-0,0502$ \\
\hline Betriebsgröße 200-1999 MA & $-0,1591 * *$ & $-0,6801$ & $-0,1445 * *$ & 0,0334 \\
\hline Betriebsgröße $2000+$ MA & $-0,1576^{* *}$ & $-0,0605$ & $-0,3305$ & $-0,0605$ \\
\hline Selbstständig & $-0,1183^{+}$ & $0,4095 * *$ & $-0,0589$ & $0,3620 * *$ \\
\hline Arbeitslosenquote & $-0,0017$ & $0,0372 * *$ & 0,0099 & $0,0408 * *$ \\
\hline Konstante & $-4,1352 * *$ & $0,1243 * *$ & $-3,3484 * *$ & $1,1871 * *$ \\
\hline
\end{tabular}

\begin{tabular}{lcccc}
\hline Signifikanzniveau: ** $=\mathrm{p}<0,01 ; *=\mathrm{p}<0,05 ;{ }^{+}=\mathrm{p}<0,1$. & \multicolumn{2}{c}{49.413} \\
Fallzahl (Personenjahre) & \multicolumn{2}{c}{70.832} & & \\
Wald $\chi^{2}$-Test (DF=38/39) & $8.014 * *$ & $2.579 * *$ & $5.107 * *$ & $3.322^{* *}$ \\
rho & 0,3880 & 0,5305 & 0,4053 & 0,4882
\end{tabular}

Abhängige Variable: 1=Arbeitszeitreduktion um mind. 4 Stunden gewünscht, $0=$ Sonstige // $1=$ Arbeitszeitausweitung um mind. 4 Stunden erwünscht, $0=$ Sonstige.

Referenzkategorien: Kinderlos mit Partner, Arbeitszeit: 30-45 Stunden, keine abgeschlossene Berufsausbildung, keine hochqualifizierte Tätigkeit/Führungsaufgaben, in der Privatwirtschaft beschäftigt, Betriebsgröße 20-199 Mitarbeiter.

Quelle: SOEP 1985-2006, eigene Berechnungen.

Väter wünschen sich seltener eine Arbeitszeitverkürzung als kinderlose Männer, jedoch sind keine signifikanten Unterschiede hinsichtlich des Wunsches zur Arbeitszeitverlängerung erkennbar. Die oben formulierte Annahme, wonach Väter aufgrund erhöhter finanzieller Belastungen ihre Arbeitszeit verlängern wollen, findet indirekte empirische Unterstützung, indem Väter signifikant häufiger einer Verkürzung der Arbeitszeit ablehnend gegenüber stehen als kinderlose Männer. Der erwartete Wunsch zur Arbeitszeitverlängerung äußert sich damit nicht in einer zunehmend positiven Differenz zwischen erwünschter und tatsächlicher Arbeitszeit, sondern darin, dass sich die negative Differenz verringert, indem sich die (niedrigere) gewünschte Arbeitzeit der (höheren) tatsächlichen Arbeitszeit annähert. Im Gegensatz zu Vätern wünschen sich Mütter mit Kindern im Vorschulalter, wie erwartet wurde, eher eine Arbeitszeitreduzierung und seltener eine Verlängerung der Arbeitszeit als kinderlose Frauen. Die Diskrepanz zwischen den oben präsentierten deskriptiven Ergebnissen und den multivariaten Befunden lässt sich dadurch erklären, dass multivariat für die tatsächliche Arbeitszeit kontrolliert wird und somit mögliche „Floor Effekte“ eliminiert werden. Ei- 
ne Ausnahme bilden allein erziehende Frauen, die signifikant häufiger eine Ausweitung sowie seltener eine Verkürzung der Arbeitszeit anstreben.

Die Koeffizienten der übrigen Kovariaten entsprechen weitgehend den formulierten Erwartungen. Mit zunehmendem Lebensalter nimmt der Wunsch zur Arbeitszeitverkürzung zu und die Neigung zur zeitlichen Intensivierung der Erwerbsarbeit ab. Die tatsächliche Arbeitszeit als auch das Erwerbseinkommen wirken sich deutlich auf den Wunsch zur Arbeitszeitverkürzung bzw. -verlängerung aus. Wie erwartet wurde, hat der Erwerbsumfang einen positiven Effekt auf den Wunsch zur Arbeitszeitverkürzung und einen negativen Effekt auf den Wunsch zur Arbeitszeitverlängerung. Ähnliches gilt für die Einkommenshöhe: Personen mit einem hohen Einkommen streben eher eine Arbeitszeitreduktion und seltener eine Verlängerung der Arbeitszeit an. Ferner zeigt sich, dass Frauen und Männer, die hochqualifizierte Tätigkeiten ausüben, stärker zu einer Arbeitszeitverkürzung neigen als andere Berufsgruppen. Entgegen unseren Erwartungen tendieren im öffentlichen Dienst beschäftigte Männer eher zu einer Reduzierung und seltener zu einer Ausweitung der Arbeitszeit. Der Zusammenhang zwischen Arbeitszeit-Mismatch und der Größe des Beschäftigungsbetriebs ist nur schwach ausgeprägt. In der Tendenz zeigt sich, dass Beschäftigte in mittleren und größeren Betrieben wie erwartet eher eine Übereinstimmung zwischen erwünschtem und faktischem Arbeitsvolumen erfahren als Personen, die in Kleinbetrieben tätig sind. Auch der Effekt der Arbeitsmarktlage auf die Übereinstimmung von Arbeitszeitpräferenzen und tatsächlichem Arbeitsvolumen bestätigt die oben formulierten Annahmen. In Zeiten hoher Arbeitslosigkeit neigt ein größerer Anteil der Erwerbstätigen zu einer Ausweitung der Arbeitszeit als in konjunkturellen Aufschwungphasen.

Der nächste Analyseschritt berücksichtigt zusätzlich den Erwerbsstatus der Partnerin bzw. des Partners. Wir unterscheiden zwischen partnerlosen Personen, kinderlosen Personen mit einem erwerbstätigen Partner, kinderlosen Personen mit einem nichterwerbstätigen Partner, Eltern mit einem erwerbstätigen Partner, Eltern mit einem nichterwerbstätigen Partner, allein Erziehenden und Personen mit erwachsenden Kindern.

Tabelle 6: Determinanten einer gewünschten Arbeitszeitreduktion / Arbeitszeitausweitung (Random-Effects Logit Regression)

\begin{tabular}{cccc}
\multicolumn{2}{c}{ Männer } & \multicolumn{2}{c}{ Frauen } \\
$\begin{array}{c}\text { Arbeitszeit- } \\
\text { reduktion }\end{array}$ & Arbeitszeit- & Arbeitszeit- re- & Arbeitszeit- \\
erwünscht & ausweitung & duktion & ausweitung \\
\hline
\end{tabular}

$\begin{array}{lllll}\text { Kein/e Partner/in } & -0,2367^{* *} & -0,2823^{* *} & -0,4734^{* *} & 0,2230^{*} \\ \text { Kein Kind, Partner NEWT } & -0,1016 & -0,0765 & -0,2421^{*} & 0,0044 \\ \text { Kind, Partner EWT } & -0,1877^{* *} & -0,2261^{*} & 0,1736^{*} & -0,4236^{* *} \\ \text { Kind, Partner NEWT } & -0,3157^{* *} & 0,0173 & -0,2468^{* *} & 0,3804^{*} \\ \text { Allein erziehend } & - & - & -0,5801^{* *} & 0,8136^{* *} \\ \text { Kind über 17 Jahre } & -0,1966^{* *} & -0,2000^{+} & -0,1322^{+} & -0,0846\end{array}$

\begin{tabular}{lcccc}
\hline Signifikanzniveau: $* *=\mathrm{p}<0,01 ; *=\mathrm{p}<0,05 ;{ }^{+}=\mathrm{p}<0,1$. & \multicolumn{2}{c}{47.789} \\
Fallzahl (Personenjahre) & 69.189 & & \\
Wald $\chi^{2}$-Test (DF=37/38) & $7.848^{* *}$ & $2.514 * *$ & $4.922^{* *}$ & $3.225^{* *}$ \\
rho & 0,3870 & 0,5321 & 0,4053 & 0,4864
\end{tabular}

Abhängige Variable: 1=Arbeitszeitreduktion um mind. 4 Stunden gewünscht, $0=$ Sonstige // $1=$ Arbeitszeitausweitung um mind. 4 Stunden erwünscht, $0=$ Sonstige EWT=Erwerbstätig; NEWT=Nichterwerbstätig.

Referenzkategorie: Kinderlose Personen mit erwerbstätigem/er Partner/in In den Modellen sind ferner alle in Tabelle 5 ausgewiesenen Kovariaten enthalten.

Quelle: SOEP 1985-2006, eigene Berechnungen. 
Hier zeigt sich, dass die Neigung zur Arbeitszeitreduktion nicht allein durch die familiale Situation, sondern ebenfalls durch den Erwerbsstatus der Partnerin oder des Partners geprägt wird, wobei jedoch starke geschlechtsspezifische Effekte zu beobachten sind. Bei Männern hat die Erwerbstätigkeit der Partnerin nur geringe Auswirkungen auf die Neigung zur Arbeitszeitverkürzung oder -reduktion. So haben kinderlose Männer mit einer erwerbstätigen Partnerin keinen stärkeren Wunsch zur Arbeitszeitreduktion als solche mit einer nichterwerbstätigen Partnerin. Ebenfalls sind nur vergleichsweise geringe Unterschiede zwischen Vätern, deren Partnerin eine Erwerbstätigkeit ausübt, und Vätern mit einer nichterwerbstätigen Partnerin zu beobachten. Hier zeigt sich immerhin, dass Erstere häufiger zu einer Arbeitszeitreduktion neigen und seltener ihren Erwerbsumfang ausdehnen möchten als Letztere. Die insgesamt schwachen Effekte des Erwerbsstatus der Partnerin auf den Wunsch zur Arbeitszeitreduktion oder -ausdehnung deuten darauf hin, dass das Arbeitsangebot der Väter in nur geringem Maße durch die Einkommensressourcen der Partnerin beeinflusst wird. Hier scheinen vielmehr gesellschaftliche Normen zu wirken, die eine Intensivierung der Erwerbsarbeit im Zuge der Familiengründung vorgeben. Dieses Ergebnis steht in Einklang mit dem Befund von Grunow et al. (2007), wonach Ehepaare im Zuge der Familiengründung - unabhängig von den jeweiligen relativen Bildungs-, Erwerbs- und Einkommensressourcen der Partner - in der Regel zu einem traditionellen Arrangement der Arbeitsteilung übergehen.

Dagegen hat bei Frauen neben der familialen Situation ebenfalls der Erwerbsstatus des Partners starke Auswirkungen auf die Neigung zur Arbeitszeitverkürzung und -ausweitung. Wie zu erkennen ist, tendieren Frauen mit einem erwerbstätigen Partner häufiger zu einer Arbeitszeitreduktion und seltener zu einer Ausdehnung der Arbeitszeit als Frauen mit einem nichterwerbstätigen Partner, wobei der Effekt der Partnererwerbstätigkeit bei Müttern deutlich stärker ausfällt als bei kinderlosen Frauen. Die interagierenden Effekte von familialer Situation und Erwerbsstatus des Partners werden besonders anhand des Vergleichs von Müttern mit einem erwerbstätigen Partner und Alleinerziehenden deutlich. Obwohl beide Personengruppen in hohem Maße Zeitkonflikte erfahren, sind grundsätzlich unterschiedliche Neigungen zur Arbeitszeitreduktion und -ausweitung erkennbar. Während Mütter mit einem erwerbstätigen Partner überdurchschnittlich häufig zu einer Verkürzung der Arbeitszeit und eher selten zu einer Arbeitszeitverlängerung tendieren, ist bei allein erziehenden Frauen ein vergleichsweise starker Wunsch zur Arbeitszeitverlängerung und eine schwache Neigung zur Arbeitszeitverkürzung erkennbar. Damit zeigt sich, dass eine hohe zeitliche Beanspruchung nicht immer den Wunsch nach kürzeren Arbeitszeiten nach sich zieht. Der Wunsch zur Arbeitszeitverkürzung ist offensichtlich nicht nur von der zeitlichen Belastung, sondern ebenfalls den hierzu notwendigen finanziellen Ressourcen abhängig.

\section{Fazit}

Bei einem beträchtlichen Anteil der Erwerbstätigen weicht das tatsächliche Arbeitsvolumen erheblich von den gewünschten Arbeitszeiten ab, wobei die überwiegende Mehrheit dieser Personen den Wunsch zur Arbeitszeitreduktion äußert. Diskrepanzen zwischen dem faktischen und dem präferierten Erwerbsumfang haben stark negative Auswirkungen auf das subjektive Wohlbefinden, was sich in einer niedrigeren Arbeits- sowie Lebenszufriedenheit äußert.

Der Befund, dass ein erheblicher Anteil der Erwerbstätigen den Erwerbsumfang reduzieren möchte, steht in Einklang mit der Erkenntnis früherer Studien, wonach eine beträchtliche Anzahl der Beschäftigten Vereinbarkeitsprobleme zwischen Arbeit und Privatleben erfährt. Allerdings ist der Zusammenhang zwischen der zeitlichen Belastung und dem Wunsch zur Arbeitszeitreduktion weniger eindeutig, als es auf den ersten Blick erscheint. Wie gezeigt wurde, wird der Wunsch zur Verringerung oder Erhöhung der Arbeitszeit wesentlich durch 
den Familien- und Haushaltskontext geprägt. Gemäß unseren Erwartungen zeigte sich, dass Mütter - insofern sie sich in einer Partnerschaft befinden - überdurchschnittlich häufig ihre Arbeitszeit verringern möchten. Dagegen neigen Väter seltener zu einer Arbeitszeitverkürzung als kinderlose Männer. Offensichtlich wirken sich die zeitlichen und finanziellen Belastungen unterschiedlich auf das Arbeitsangebot von Müttern und Väter aus: Während die Arbeitszeitwünsche der Mütter eher durch die zeitlichen Restriktionen geprägt werden, wird der gewünschte Erwerbsumfang der Väter stärker durch die finanziellen Bedürfnisse des Haushalts beeinflusst. Gleichzeitig scheinen gesellschaftliche Erwartungen die Arbeitszeitwünsche der Väter zu prägen, was sich darin zeigt, dass Väter ihr Arbeitsangebot ungeachtet der ökonomischen Haushaltsressourcen und der Erwerbskonstellation des Haushalts festlegen.

Zeitkonflikte initiieren nicht zwangsläufig einen Wunsch nach kürzeren Arbeitszeiten, wie auch der Wunsch nach kürzeren Arbeitszeiten nicht unbedingt auf Zeitkonflikte schließen lässt. So äußern bestimmen Personengruppen, die generell überdurchschnittlich häufig unter Zeitkonflikten leiden, wie Väter in Doppelverdienerhaushalten oder allein erziehende Mütter (siehe Künzler et al. 2001), eher selten den Wunsch nach kürzeren Arbeitszeiten. In einem besonderen Spannungsfeld befinden sich allein Erziehende, die zum einen hohen familialen Belastungen ausgesetzt sind, zum anderen jedoch offenbar aufgrund ihrer oftmals ungünstigen finanziellen Situation eine mit der Arbeitszeitverringerung einhergehende Einkommensreduktion nicht verkraften können. Dagegen möchten kinderlose Frauen und Männer in einer Partnerschaft - die eher geringe Zeitkonflikte erfahren dürften, aber über ein relativ hohes Haushaltseinkommen verfügen - besonders häufig ihre Arbeitszeit reduzieren.

Studien zur Vereinbarkeit von Familie und Beruf fordern in der Regel eine Verkürzung der Arbeitszeit als ein probates Mittel zur Verminderung von Zeitkonflikten. Die vorliegende Untersuchung macht allerdings deutlich, dass der Zusammenhang zwischen Zeitkonflikten und den Arbeitszeitwünschen wesentlich komplexer ist als häufig angenommen wird. Ob Erwerbstätige ihren Erwerbsumfang reduzieren oder erhöhen möchten, hängt offenbar von ihrer Arbeitszeit und ihren familialen Verpflichtungen, aber auch von finanziellen Ressourcen und verinnerlichten gesellschaftlichen Normen ab.

\section{Literatur}

Baaijens, Christine / Anneke van Doorne-Huiskes / Joop Schippers (2005): Do Dutch employees want to work more or fewer hours than they actually do?, in Bram Peper / Anneke van Doorne-Huiskes / Laura den Dulk (Hrsg.), Flexible working and organisational change. The integration of work and personal life, Cheltenham, S. 201-221.

Bauer, Frank / Hermann Groß /Klaudia Lehmann / Eva Munz (2004): Arbeitszeit 2003. Arbeitszeitgestaltung, Arbeitsorganisation und Tätigkeitsprofile, Köln: ISO-Institut.

Bauer, Frank / Eva Munz (2005): Arbeitszeiten in Deutschland: 40plus und hochflexibel, in: WSI Mitteilungen 58, S. 40-48.

Beckmann, Petra / Birgit Kempf (1996): Arbeitszeiten und Arbeitswünsche von Frauen in West- und Ostdeutschland, in: Mitteilungen aus der Arbeitsmarkt- und Berufsforschung 29, S. 388-408.

Berg, Peter / Arne L. Kalleberg / Eileen Appelbaum (2003): Balancing work and family: the role of highcommitment environments, in: Industrial Relations 42, S. 168-188.

Bielenski, Harald / Gerhard Bosch / Alexandra Wagner (2002): Wie die Europäer arbeiten wollen. Erwerbs- und Arbeitszeiten in 16 Ländern, Frankfurt / Main.

Blau, Francine D. / Lawrence M. Kahn (2007): Changes in the labor supply behaviour of married women: 1980-2000, in: Journal of Labor Economics 25, S. 393-438. 
Böheim, Rene / Mark P. Taylor (2004): Actual and preferred working hours, in: British Journal of Industrial Relations 42, S. 149-166.

Bosch, Gerhard (2000): Arbeitszeit und Arbeitsorganisation. Zur Rolle von Produkt- und Arbeitsmärkten im internationalen Vergleich, in: Arbeit 9, S. 175-190.

Devereux, Paul J. (2004): Changes in relative wages and family labor supply, in: Journal of Human Resources 39, S. 696-722.

Drago, Robert / Yi-Peng Tseng / Marc Wooden (2005): Usual and preferred working hours in couple households, in: Journal of Family Studies 11, S. 46-61.

Franz, Wolfgang (2003): Arbeitsmarktökonomik, Berlin.

Galinsky, Ellen / Stacy S. Kim / James T. Bond (2001): Feeling overworked: When work becomes too much, New York: Families and Work Institute.

Garhammer, Manfred (2004): Auswirkungen neuer Arbeitsformen auf Stress und Lebensqualität, in Bernhard Badura / Henner Schellschmidt / Christian Vetter (Hrsg.), Fehlzeiten-Report 2003. Wettbewerbsfaktor Work-Life-Balance. Zahlen, Daten, Analysen aus allen Branchen der Wirtschaft, Berlin, S. $45-74$.

Glass, Jennifer L. / Sarah Beth Estes (1997): The family responsive workplace, in: Annual Review of Sociology 23, S. 289-313.

Grözinger, Gerd / Wenzel Matiaske / Verena Tobsch (2008): Arbeitszeitwünsche, Arbeitslosigkeit und Arbeitszeitpolitik, in: WSI Mitteilungen 61, S. 92-99.

Grunow, Daniela / Florian Schulz / Hans-Peter Blossfeld (2007): Was erklärt die Traditionalisierungsprozesse häuslicher Arbeitsteilung im Eheverlauf: soziale Normen oder ökonomische Ressourcen?, in: Zeitschrift für Soziologie 36, S. 162-181.

Hamermesh, Daniel S. (1996): Workdays, workhours and work schedules. Evidence for the United States and Germany, Kalamazoo: W. E. Upjohn Institute for Employment Research.

Jacobs, Jerry A. / Kathleen Gerson (2001): Overworked individuals or overworked families?, in: Work and Occupations 28, S. 40-63.

Jacobs, Jerry A. / Kathleen Gerson (2004): The time divide. Work, family, and gender inequality, Cambridge.

Jürgens, Kerstin (2005): Kein Ende von Arbeitszeit und Familie, in: Anina Mischau / Mechtild Oechsle (Hrsg.), Arbeitszeit - Familienzeit - Lebenszeit: Verlieren wir die Balance? (Sonderheft 5 der Zeitschrift für Familienforschung), Wiesbaden, S. 34-53.

Künzler, Jan / Wolfgang Walter / Elisabeth Reichart / Gerd Pfister (2001): Gender division of labour in unified Germany. WORC Report, Tilburg: European Network on Policies and the Division of Unpaid and Paid Work.

Lehndorff, Steffen (2003): The Long Good-Bye? Tarifvertragliche Arbeitszeitregulierung und gesellschaftlicher Arbeitszeitstandard, in: Industrielle Beziehungen 10, S. 273-295.

Lundberg, Shelly (1988): Labor supply of husbands and wives: a simulation equations approach, in: Review of Economics and Statistics 70, S. 224-235.

Madalla, Gangadharrao S. (1987): Limited dependent variable models using panel data, in: Journal of Human Resources 22, S. 307-338.

Moen, Phyllis / Donna Dempster-McClain (1987): Employed parents: role strain, work time, and preferences for working less, in: Journal of Marriage and the Family 49, S. 579-590.

Morissette, René / Feng Hou (2008): Does the labour supply of wives respond to husband's wages? Canadian evidence form micro data and grouped data, in: Canadian Journal of Economics 41, S. $1185-1210$.

Pollmann-Schult, Matthias / Martin Diewald (2007): Auswirkungen der Familiengründung auf den Berufsverlauf von Männern, in: Kölner Zeitschrift für Soziologie und Sozialpsychologie 59, S. 440-458. 
Reynolds, Jeremy (2005): In the face of conflict: work-life conflict and desired work hour adjustments, in: Journal of Marriage and the Family 67, S. 1313-1331.

Reynolds, Jeremy (2006): Pursuing preferences: the creation and resolution of work hour mismatches, in: American Sociological Review 71, S. 618-638.

Rürup, Bert / Sandra Gruescu (2005): Familienorientierte Arbeitszeitmuster - Neue Wege zu Wachstum und Beschäftigung, Berlin: Bundesministerium für Familie, Senioren, Frauen und Jugend.

Schulz, Florian / Hans-Peter Blossfeld (2006): Wie verändert sich die häusliche Arbeitsteilung im Eheverlauf? Eine Längsschnittstudie der ersten 14 Ehejahre in Westdeutschland, in: Kölner Zeitschrift für Soziologie und Sozialpsychologie 58, S. 23-49.

Stamper, Christina L. / Linn Van Dyne (2001): Work status and organizational citizenship behavior: a field study of restaurant employees, in: Journal of Organizational Behavior 22, S. 517-536.

Vaskovics, Laszlo A. / Harald Rost / Doris Rosenkranz (2000): Was machen junge Väter mit ihrer Zeit? Die Zeitallokation junger Ehemänner im Übergang zur Elternschaft. ifb-Forschungsbericht Nr. 2, Bamberg: Staatsinstitut für Familienforschung an der Universität Bamberg.

Wagner, Alexandra (2001): Entgrenzung der Arbeit und der Arbeitszeit, in: Arbeit 10, S. 365-378.

Wagner, Gert G. / Joachim R. Frick / Jürgen Schupp (2007): The German Socio-Economic Panel Study (SOEP) - scope, evolution and enhancements, in: Schmollers Jahrbuch - Journal of Applied Social Science Studies 127, S. 139-169.

Wooldridge, Jeffrey M. (2000): Introductory econometrics: a modern approach, Mason.

Dr. Matthias Pollmann-Schult

Universität Bielefeld

Fakultät für Soziologie

Postfach 100131

33501 Bielefeld

matthias.pollmann-schult@uni-bielefeld.de 